\title{
A Case Series of Budd-Chiari Syndrome in Nigerian Patients: Diagnosis, Associations, Management, and Outcomes
}

\author{
Hammed Ninalowo ${ }^{1}$ Aderemi Oluyemi ${ }^{2} \quad$ Omodele Olowoyeye $^{3} \quad$ Abisoye Ajayi $^{3}$ \\ ${ }^{1}$ Interventional Radiology, IRDOC Interventional Radiology \\ Consulting Limited, Euracare Multispecialty Hospital, Victoria \\ Island, Lagos, Nigeria \\ 2Department of Gastroenterology, Endoscopy and Hepatology, \\ ReMay Consultancy and Medical Services, Ikeja, Lagos State, \\ Nigeria \\ ${ }^{3}$ Department of Radiodiagnosis, Lagos University Teaching Hospital, \\ Idi-Araba, Lagos, Nigeria \\ Address for correspondence Aderemi Oluyemi, Department of \\ Gastroenterology, Endoscopy and Hepatology, ReMay Consultancy \\ and Medical Services, No. 24, Omotayo Ojo Street, Ikeja, Lagos \\ State 101212, Nigeria (e-mail: remioluyemi@yahoo.com).
}

\begin{abstract} Keywords

- Budd-Chiari syndrome

- Nigeria

- interventional radiology

- transjugular intrahepatic postosystemic stent-shunt

- venoplasty

Budd-Chiari syndrome (BCS) is defined as hepatic outflow obstruction regardless of the cause or level of obstruction-from small hepatic veins to the opening of the inferior vena cava in the right atrium. BCS could be primary or secondary. Very few cases of this syndrome have been reported in Nigeria and there is no local clinical series documenting the noninvasive and invasive imaging findings and importance of interventional radiology techniques in its diagnosis and management.

We report three cases of patients diagnosed with BCS in Lagos, Nigeria. We hope this work will raise awareness about the condition and its associations and show that much can be done to establish diagnosis and care with minimally invasive techniques in our resource-limited environment.
\end{abstract}

\section{Introduction}

Budd-Chiari syndrome (BCS) derives its name from the internist George Budd and the pathologist Hans Chiari who described the disease. This syndrome could be primary or secondary. ${ }^{1,2}$ Primary BCS occurs when there is an intraluminal thrombus or obstruction to the hepatic venous outflow (HVO) anywhere from the small hepatic veins to the opening of the internal vena cava (IVC) into the right atrium. When HVO obstruction is due to compression or invasion by benign or malignant extravascular lesions, such as a cyst or hepatocellular cancer, or secondary to cardiac or pericardial diseases, the term "secondary BCS" is more appropriately used. ${ }^{1,2}$ It should be noted, however, that

published online October 4, 2021
DOI https://doi.org/

$10.1055 / \mathrm{s}-0041-1731975$

ISSN 2581-9933 sinusoidal obstruction syndrome (which was previously known as veno-occlusive disease) is not included in this BCS definition. ${ }^{1,2}$

In the vast majority of cases, at least one underlying prothrombotic factor is documented to be associated with BCS. ${ }^{3}$ Therapeutic options include medical management (e.g., use of diuretics, anticoagulants/thrombolytics), interventional procedures (such as insertion of transjugular intrahepatic portosystemic stent-shunt [TIPS]), and surgical (e.g., portosystemic shunting and liver transplantation). ${ }^{3}$

The global estimated incidence and prevalence of BCS are 1 per million per year and 11 per million, respectively. ${ }^{4}$ The rarity of the disease in Nigeria is underlined by the fact that only a handful of BCS cases have been documented. ${ }^{5-7}$ (c) 2021. Indian Society of Gastrointestinal and Abdominal Radiology. This is an open access article published by Thieme under the terms of the Creative Commons Attribution-NonDerivative-NonCommercial-License, permitting copying and reproduction so long as the original work is given appropriate credit. Contents may not be used for commercial purposes, or adapted, remixed, transformed or built upon. (https://creativecommons.org/licenses/by-nc-nd/4.0/).

Thieme Medical and Scientific Publishers Pvt. Ltd. A-12, 2nd Floor, Sector 2, Noida-201301 UP, India 


\section{Case Reports}

\section{Case 1}

The first patient is a 17-year-old girl who presented with a 3-year history of refractory ascites managed with high-volume paracentesis (15 L/2 week) and albumin infusions. She had no history of alcohol ingestion and her hepatitis screens were negative. On physical examination, she appeared cachectic, with evidence of diffuse muscle wasting and grossly distended abdomen with an associated fluid-filled umbilical hernia. At presentation, the model for end-stage liver disease (MELD) score was 11, and her BCS-TIPS prognostic index (BCS-TIPS PI) score was 5.6. Cross-sectional imaging showed hepatomegaly with nutmeg appearance of the liver, absence of the hepatic veins, narrowing of the IVC, and massive ascites with associated umbilical hernia filled with fluid. Subsequently, she underwent a direct transcaval portosystemic shunt (DIPS) which is a direct transhepatic connection from the IVC to the portal vein. It is a modified technique of TIPS in which a transhepatic stent is placed into portal system via a patent hepatic vein to decompress portal vein pressure. A side-by-side IVC stent was also placed at this time, due to presence of marked narrowing of the proximal IVC. This, to the best of our knowledge, was the first time an endovascular portosystemic shunt was successfully performed in Nigeria. ( -Fig. 1A, B) provide details about the intraoperative findings of successful intrahepatic tract connection of the IVC to the left portal vein, significantly narrowed IVC, and the successful placement of side-by-side portosystemic shunt/IVC stents with resolution of IVC stenosis. Success of the procedure was highlighted by the marked improvement in postprocedure portosystemic gradient from 26 to 6 . As at the time of preparing this report, a clear underlying etiology for her BCS had not been found. Since the procedure, she has had a significant improvement in volume of ascites from 15 L fortnightly to 5 L every 6 weeks. Despite lack of complete
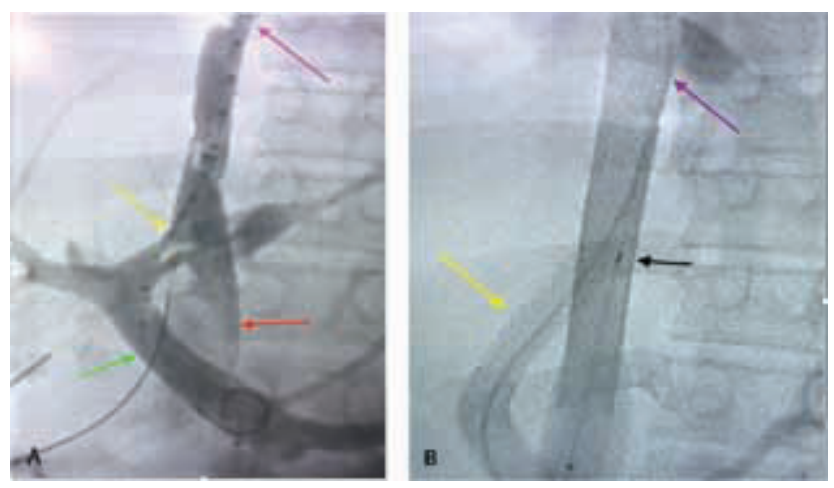

Fig. 1 (A) Intraprocedural venogram with simultaneous injection from the inferior vena cava (IVC) and portal vein (PV) shows pigtail catheter (yellow arrow) bridging the IVC to left portal vein tract. Also shows a patent main PV (green arrow), severe stenosis of the IVC near the right atrial junction and expected drainage location of the hepatic veins (purple arrow), and dilation of the distal IVC (red arrow). (B) Completion venogram with simultaneous injection from the inferior vena cava (IVC) and PV shows the direct portosystemic shunt stent graft (yellow arrow), side-by-side IVC stent, and resolution of IVC narrowing post stent (purple arrow). resolution of ascites, she has had a significant improvement in quality of life, with return to school and daily activities.

\section{Case 2}

A 60-year-old Nigerian man presented at the hepatology clinic with features of advanced chronic liver disease. The symptoms had started some 6 months earlier with feeling of malaise, loss of appetite, and unintentional weight loss. He had no history of alcohol ingestion and his test results showed negative hepatitis screens, mild elevations of transaminases, and an ultrasound (US) scan showing an enlarged liver with nodular, heterogenous parenchymal echogenicity. An initial blind liver biopsy was also reported as normal and, thus, he was being managed for chronic liver disease of unknown etiology. However, his condition had rapidly deteriorated over more recent months with worsening of initial symptoms, new-onset right-sided abdominal pain and abdominal distention, jaundice, icterus and new anemia. Repeat US-guided biopsy (targeted at one of the hepatic nodules) revealed findings in keeping with an acute cause of vascular outflow obstruction with large areas of zonal hemorrhagic necrosis (up to one-third of the parenchymal sampled) and lobular disarray with patchy sinusoidal congestion. A repeat US scan showed additional findings of ascites, narrowing of the right hepatic vein, with nonvisualization of the left and middle hepatic veins. A Doppler interrogation was suggestive of portal hypertension with increased hepatic resistive indices. A computed tomography (CT) study revealed "nutmeg appearance" of the liver, which is a described by a diffuse heterogenous and nodular enhancement pattern of the liver (-Fig. 2). He underwent hepatic venogram and pressure measurements which showed evidence of hepatic terminal venular disease, narrowing of the right hepatic vein near the IVC confluence, and narrowing of the IVC ( - Fig. 3 ). There was evidence of severe portal hypertension with portosystemic gradient of $20 \mathrm{mmHg}$. This was concordant with biopsy findings of hepatic necrosis and nutmeg appearance

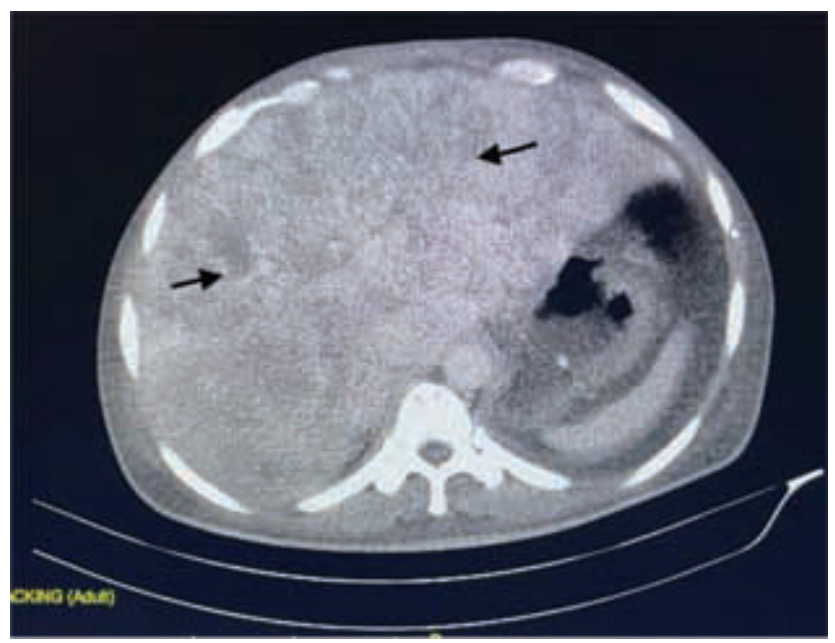

Fig. 2 Computed tomography scan of the abdomen: In the venous phase, it shows diffuse heterogenous and nodular enhancement pattern of the liver (black arrows), classically known as nutmeg appearance. The hepatic veins (not shown) were found to be patent. 

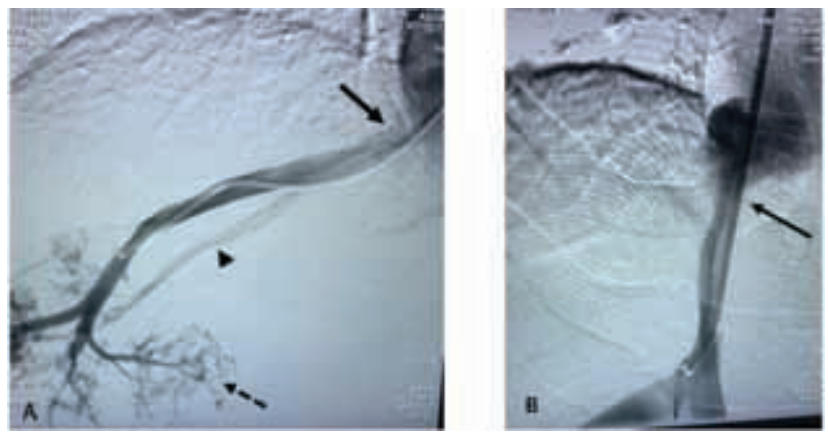

Fig. 3 (A) Moderate stenosis of the right hepatic vein near the inferior vena cava (IVC) insertion (black arrow). This is masked by an overlapping parallel collateral filling vein (arrowhead). The hepatic terminal venules have an abnormal web-like appearance with venovenous connections (broken arrow). (B) A smooth long segment narrowing of the intrahepatic segment of the IVC.

of the liver on cross-sectional imaging and led to a diagnosis of BCS. At that time, his BCS-TIPS index was 6.43 and MELD score was 22. Further testing revealed hemoglobinuria along with elevated reticulocyte counts and serum lactate dehydrogenase (LDH) levels, with eventual conclusion of paroxysmal nocturnal hemoglobinuria as confirmed by flow cytometry (marked reduced levels of both CD 59 and CD 55 on red blood cells). His condition continued to deteriorate, with repeat evaluation showing a MELD score of 30 and BCS-TIPS index of 7.56. Due to continued decompensation, we advised withholding any intervention at the material time. He died shortly from multiorgan failure.

\section{Case 3}

Our third patient is a 25-year-old woman with a previous history of polycythemia vera (PV) which was diagnosed 5 years prior. She had been placed on warfarin, hydroxyurea, and aspirin but stopped these medications 2 years prior due to intolerable side effects. At presentation, her predominant complaint was right-sided upper abdominal pain. CT scan revealed massive hepatomegaly and focal narrowing of the intrahepatic IVC. A US scan showed nonpatency of the main hepatic veins. An assessment of BCS was made secondary to intrahepatic IVC stenosis and nonvisualization of the hepatic veins. Her Rotterdam score was 0.67 (class I) which predicts an excellent intervention-free survival. Hepatic venography, IVC angioplasty, and a liver biopsy were performed in the same sitting. - Fig. 3 pictorially depicts the intraoperative findings on venography and the IVC dimensions pre and post venoplasty. Pressure measurements were taken with IVC predilation pressure of $14 \mathrm{mmHg}$ and right atrium mean $1 \mathrm{mmHg}$ (gradient 13). Luminal caliber of the IVC improved and the pressure gradient across the narrowing reduced with IVC prestenotic pressure $18 \mathrm{mmHg}$ and right atrium $15 \mathrm{mmHg}$ (gradient 3) (-Fig. 4). It is unclear exactly why the post venoplasty prestenotic IVC segment pressure was higher; however, the gradient significantly changed from 13 to 3 . The ostium of the main hepatic vein was not identified; we did access the accessory hepatic vein which was enlarged and likely drained the enlarged caudate lobe
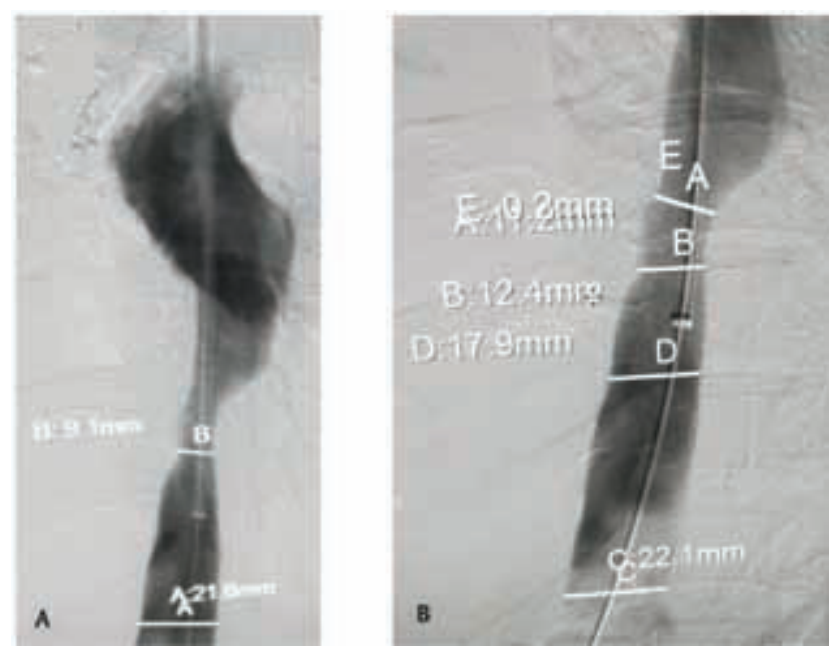

Fig. 4 Angioplasty of the inferior vena cava. (A) Pre angioplasty venogram showing the pre-stenotic segment (line $A=21 \mathrm{~mm}$ in cross-sectional diameter) and stenotic segment (line $B=9 \mathrm{~mm}$ in cross-sectional diameter) prior to angioplasty. (B) Post angioplasty venogram showing the pre-stenotic segment (Line $\mathrm{C}=22.1 \mathrm{~mm}$ in cross-sectional diameter). It also shows the much improved caliber of the previously stenotic segment (Lines $D=17.9 \mathrm{~mm}$ and $B=12.4 \mathrm{~mm}$ \& $E A=11.2 \mathrm{~mm}$ ). There was thus a marked decrease in gradient across this segment from 13 to 3 .

and majority of the liver. Liver biopsy showed sinusoidal dilatation with congestion, regenerative nodules, and ductular reaction-all of which was in keeping with our BCS diagnosis. Currently she is being managed for PV by the hematology team and is on long-term anticoagulation.

\section{Discussion}

Diagnosis of BCS can be challenging in any environment. Its rarity, especially in places such as our locality, demands a high clinical index of suspicion and though the classic triad of abdominal pain, ascites, and hepatomegaly is observed in the vast majority of patients with BCS, these are nonspecific. ${ }^{5,8}$ Doppler US scan is regarded as the initial technique of choice for diagnosis and offers a high sensitivity and specificity. ${ }^{9,10}$

Findings on US scan include hepatomegaly with heterogenous echotexture of the liver. The caudate lobe is enlarged in most patients, which is explained by drainage of the caudate lobe vein into the IVC directly. On color Doppler US, there is nonvisualization of the hepatic veins. A fibrous cord replacing the hepatic veins may be seen. Other findings may include reversal of flow within the portal vein and increased resistive index within the hepatic artery. In the chronic setting, features of portal hypertension and chronic hepatic regeneration may also be seen including ascites and regenerative nodules. On CT or magnetic resonance imaging (MRI), findings include inability to identify hepatic veins, early enhancement of the caudate lobe and central liver around the IVC, delayed enhancement of the peripheral liver with accompanying central low density (flip-flop appearance), and inhomogeneous mottled liver (nutmeg liver). In the chronic phase, there is caudate lobe enlargement and 
atrophy of the peripheral liver in affected areas. Angiographic findings include complete occlusion of hepatic vein(s), with or without focal stenosis of the intrahepatic IVCS and spider web appearance of the intrahepatic collateral veins. The heterogeneity of imaging service providers in Nigeria, which includes many unlicensed ultrasonographers providing unsupervised services, can make proper diagnosis difficult and in most instances result in non- or delayed diagnosis. It must be noted at this juncture that liver biopsy is no longer considered necessary for a definitive diagnosis. ${ }^{2,9}$ DeLeve and colleagues have summarized the diagnostic features of BCS on Doppler US scan. ${ }^{2}$ The intravascular images of all three cases presented in this article detail the venographic findings ranging from terminal venule obstruction to hepatic vein occlusion to IVC stenosis. This by no means suggests that these beneficial tools are readily available in the country. In two of the cases (2 and 3), supportive tissue evidence was obtained from histological reports of liver biopsies.

There are striking geographical differences in the causative factors of BCS, according to documented reports. In Asia, idiopathic membranous obstructions (webs) in the IVC are the most prevalent cause of BCS while in the West, BCS is usually a hepatic vascular complication of an underlying prothrombotic condition. ${ }^{9,11}$ But in China, the factors that predispose to these thrombotic events are unknown in the majority of cases. ${ }^{12}$ Overall, the underlying causes are not determined in about a third of BCS cases. ${ }^{4}$ This fact is illustrated in this clinical series presentation as the underlying disorder in case 1 is unknown, case 2 was diagnosed with paroxysmal nocturnal hemoglobinuria (an acquired prothrombotic condition), and case 3 was polycythemia vera (a myeloproliferative disease). For the management of BCS to be complete, the care of these associated conditions must be done concurrently. Thus, the management of BCS requires input from various disciplines including hematology, hepatology, and interventional radiology (IR). Therapeutic options for BCS include medical management with anticoagulation therapy and therapy for the underlying condition; decompressing therapies such as recanalization strategies (thrombolytic therapy, stenting, and angioplasty); surgical shunting and TIPS; and, lastly, liver transplantation (LT). ${ }^{9}$

Thrombolytic therapy alone appears to be of little effect for those with more significant thrombotic disease while surgical portosystemic shunting has been largely abandoned because of its abysmal inability to show any survival benefit., ${ }^{3,9}$ Medical therapy alone appears to control the disease in a small percentage of patients with mild disease..$^{13}$ This was the method adopted in the case report from Calabar, Nigeria, to good effect. ${ }^{5}$ This, however, is not enough in a majority of cases. The current approach to therapeutic intervention favors the deployment of minimally invasive techniques offered by interventional radiology. ${ }^{13}$ The goal of minimally invasive interventions are either to relieve narrowing of an occluded hepatic vein or IVC using angioplasty/stenting or the creation of a portosystemic shunt to bypass the congested liver or obstructed hepatic veins. Two of these are demonstrated in this case series. Case 1 had total absence of the hepatic veins and atresia of the intrahepatic IVC. The patient was successfully treated with DIPS/IVC stent placement. Case 3 had successful angioplasty for a significantly stenosed part of the intrahepatic IVC with near normalization of the postdilation pressures across this stenotic segment. The two cases where successful intervention was possible highlight the fact that much can yet be done in spite of constraints in this locality. It also underlines the importance of IR as a vital partner in the field of hepatology, especially as it relates to vascular hepatic diseases. The paltry number of centers that offer IR service among our over 150 million population is sad indeed.

As it relates to prognosis and outcomes, multiple factors have been shown to decide this. Composite prognostic scoring systems have been devised specifically for BCS. ${ }^{14}$ The Rotterdam index was validated to be excellent in predicting intervention-free survival while the BCS-TIPS prognostic index (PI) score was strongly associated with survival in patients who received TIPS and had a discriminative capacity, which was superior to the Rotterdam score. ${ }^{3}$ Case 1 had a successful shunt procedure and is still alive and well as at the time of publishing this article-the patient's favorable BCS-TIPS score at presentation for the procedure had been pointed out previously. Our second case had presented with a class III Rotterdam index score of 2.5 and BCS-TIPS index of 7.56. This has been shown (and validated) to be associated with a poor intervention-free prognosis and 5-year survival of less than $45 \%{ }^{3,15}$ The condition was further underlined as precarious as the patient's MELD score was advanced as well. He passed on less than a month after the diagnosis was established. The last case presented had marked improvement after venoplasty and reintroduction of medical therapy for polycythemia vera. At diagnosis, the patient's favorable class I Rotterdam score was much cause for encouragement in her case and the outcome was satisfactory as well. Though the poor performance of BCS-specific prognostic indices when applied to a given patient has been noted, outcomes in our cases appear to buck this trend. ${ }^{14}$

\section{Conclusion}

The series presented here has documented three cases of the rare diagnosis of BCS in patients in Southwest Nigeria. It details the importance of having a high index of suspicion and the appropriate expertise in radiological diagnosis. Also, it highlights the importance of a multidisciplinary approach to both diagnosis and treatment. And lastly, the article underscores the important role of interventional radiology in the management of patients who could benefit from endovascular interventions. We hope that this article will raise awareness about the condition and stimulate policy to address the local deficiencies, especially in the availability of interventional radiology.

Conflict of Interest

None declared. 


\section{References}

1 Janssen HL, Garcia-Pagan JC, Elias E, Mentha G, Hadengue A, Valla DC; European Group for the Study of Vascular Disorders of the Liver. Budd-Chiari syndrome: a review by an expert panel. J Hepatol 2003;38(3):364-371

2 DeLeve LD, Valla DC, Garcia-Tsao G; American Association for the Study Liver Diseases. Vascular disorders of the liver. Hepatology 2009;49(5):1729-1764

3 Seijo S, Plessier A, Hoekstra J, et al; European Network for Vascular Disorders of the Liver. Good long-term outcome of Budd-Chiari syndrome with a step-wise management. Hepatology 2013;57(5):1962-1968

4 Li Y, De Stefano V, Li H, et al. Epidemiology of Budd-Chiari syndrome: a systematic review and meta-analysis. Clin Res Hepatol Gastroenterol 2019;43(4):468-474

5 Kooffreh-Ada M, Chukwudike E, Kajogbola G, Eghwubare F, Okonkwo U, Ngim O. Budd Chiari syndrome: an unexpected diagnosis in a gastroenterology practice in Calabar, Nigeria. Glob J Pure Appl Sci 2019;25:103-107

6 Shah SP, Sandhu R, Mitchell S, Larkin G. Visual loss precluding acute onset of Budd-Chiari syndrome. Eye (Lond 2006;20(9):1097-1099

7 BusinessWireIndia. Fortis Hospital Noida performs India's first living donor liver transplant, on a Nigerian patient suffering from Budd-Chiari Syndrome (BCS) with fully obstructed inferior vena cava (IVC). Accessed July 6, 2020 at: https:// www.businesswireindia.com/fortis-hospital-noida-performsindia's-first-living-donor-liver-transplant-on-a-nigerian-patient-suffering-from-budd-chiari-syndrome-bcs-with-fully-obstructed-inferior-vena-cava-ivc-43000.html.

8 Goel RM, Johnston EL, Patel KV, Wong T. Budd-Chiari syndrome: investigation, treatment and outcomes. Postgrad Med J 2015;91(1082):692-697

9 Martens P, Nevens F. Budd-Chiari syndrome. United European Gastroenterol J 2015;3(6):489-500

10 Bolondi L, Gaiani S, Li Bassi S, et al. Diagnosis of Budd-Chiari syndrome by pulsed Doppler ultrasound. Gastroenterology 1991;100(5 Pt 1):1324-1331

11 De BK, De KK, Sen S, et al. Etiology based prevalence of BuddChiari syndrome in eastern India. J Assoc Physicians India 2000;48(8):800-803

12 Dang X, Li L, Xu P. Research status of Budd-Chiari syndrome in China. Int J Clin Exp Med 2014;7(12):4646-4652

13 Plessier A, Sibert A, Consigny Y, et al. Aiming at minimal invasiveness as a therapeutic strategy for Budd-Chiari syndrome. Hepatology 2006;44(5):1308-1316

14 MacNicholas R, Olliff S, Elias E, Tripathi D. An update on the diagnosis and management of Budd-Chiari syndrome. Expert Rev Gastroenterol Hepatol 2012;6(6):731-744

15 Darwish Murad S, Valla DC, de Groen PC, et al. Determinants of survival and the effect of portosystemic shunting in patients with Budd-Chiari syndrome. Hepatology 2004;39(2):500-508 